\title{
Non destructive testing of works of art by stimulated infrared thermography and terahertz analysis
}

\author{
J.L Bodnar*, J.J Metayer**, K. Mouhoubi* et V. Detalle*** \\ * GRESPI/ ECATHERM, UFR Sciences Exactes et Naturelles, BP 1039, 51687 Reims cedex 02, France \\ ** VISIOM, Parc de haute Technologie, 13 rue Alexis de Tocqueville, SILIC 41, 92182 Antony, France \\ *** Laboratoire de Recherche des Monuments Historiques, 29 avenue du Paris, 77420 Champs sur Marne, \\ France
}

\begin{abstract}
The improvement of technologies and the increase in the need for safety in the airports lead to the development of non-destructive testing devices using terahertz waves. These waves present indeed, the advantage of being on the one hand relatively penetrating. They have the advantage in addition of not being ionizing. It is thus potentially of an interesting contribution in the field of the non destructive testing.

With the help of the VISIOM Company, we then approached the possibilities of this new method of analysis for the assistance to the restoration of the works of art.

We show here, on the one hand that the method allow, like the photothermal method, the detection of defects located in murals paintings or marquetries.

We show in addition that the method allows the detection of defects potentially masked by other defects, which does not allow stimulated infra-red thermography.

We show in the third time, that the method does not seem sensitive to the various pigments constituting the pictorial layer, which constitutes also an advantage compared to the photothermal method.

We show finally, that the terahertz method is limited as regards analysis of low-size defects, which is not the case of stimulated infra-red thermography.
\end{abstract}

\section{Introduction}

The frequency band of the terahertz waves is extending approximately between $100 \mathrm{GHz}$ and $30 \mathrm{THz}$. That corresponds to the wavelengths varying approximately between $0,1 \mathrm{~mm}$ and $3 \mathrm{~mm}$. This band is intermediate between the microwaves and the optical frequencies of the infra-red. Until now, it was not really explored because of the absence of adapted detector and radiation sources. The need for safety increasing in air transports and the improvement of technologies make that this spectral band was these last years studied again [1-6]. Thus new sources are now available in the scientific laboratories. We can cite on the one hand, the sources implementing quantum cascade lasers. We can cite in addition, the Gunn diodes. In the same way, various types of detectors are available. We can cite traditional antennas. We can cite also the terahertz / thermal infrared transducers. In spite of the limited lateral resolution of the terahertz method (typically $200 \mu \mathrm{m}$ for a wavelength of $2 \mathrm{~mm}$ ), this frequency band present many advantages. First of all, like the infrareds, the terahertz waves can be worked optically. Then, like the microwaves, they have an important capability of penetration. They are not ionizing. Finally, the terahertz waves are, via a transducer, detectable using a thermal detector, like an infra-red camera of thermography. Because of theses advantages, we decided to develop a study to approach the possibilities of this spectral band for the assistance of work of art. The experimental device used is the "Provision 100" of VISIOM Company. Today, it is a device classically used in the airports. Using this industrial 
device, we analyzed various types of works of art. We present here examples of results obtained within this framework. We compare them with those obtained by traditional stimulated infra-red thermography [7-10].

\section{The experimental system used: The "PROVISIOM 100 “}

The experimental system used for this study is the "PROVISION 100" of "VISIOM" Company (figure 1a). This system is a terahertz scanner classically used in the airports. It makes it possible to detect firearm, explosives, knives, drugs... It contains approximately 400 couples Gunn diodes/aerials. These sources of emission/reception constitute two bands of analysis of approximately $2 \mathrm{~m}$ height. First is located in front face of the studied sample. The second is located in rear face of the studied sample. The complete analysis of the studied sample is obtained by a rotation of 180 degrees of this couple of bands of analyses (figure $1 \mathrm{~b}$ )

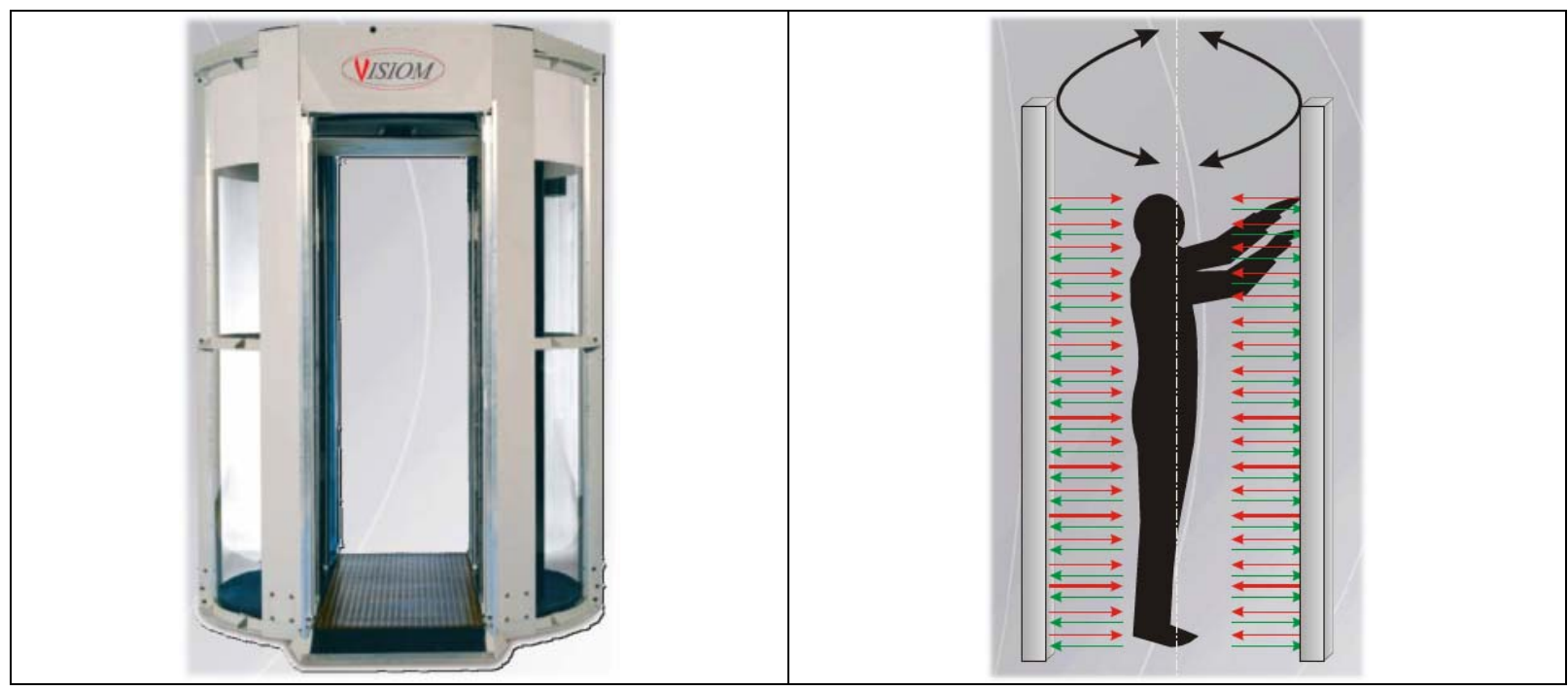

Figure 1: The terahertz system: “PROVISIOM 100” System (on the left) and its operating principle (on the right)

The frequency band used by "PROVISIOM 100" system is [24 GHZ- 30 GHZ]. The duration of a complete analysis is equal to $2 \mathrm{~s}$. This duration is really very small. It is an advantage for this non destructive testing method. The density of power implemented by "PROVISIOM 100 ” system is approximately equal to $68 \mu \mathrm{W} / \mathrm{cm} 2$ (for a distance equal to $1 \mathrm{~m}$ ). This value is also very small. It is 10000 times lower than that measured near a portable telephone. This value gives a non destructive character on the method. Finally, the "PROVISIOM 100" system uses on the one hand, the properties of absorption of the terahertz waves presented by the polar molecules, such as water (atmosphere) or metals. It uses in addition, the properties of transparency (on a typical thickness of $1 \mathrm{~cm}$ ) of the textiles (clothing), wood, Teflon, plastics, paper, ceramics, concrete... The analysis given by "PROVISIOM 100" system consists in building images of terahertz holographic interferometry of studied sample.

\section{Examples of experimental results obtained}

\section{1) Detection of delaminations in a Lime / plaster sample}


First, we want to study the possibilities of the terahertz waves for the detection of delamination located in an academic mural painting. To this end, we have realized a test sample. We have used the primitive Italians technique. We made a plaster block. We covered it with a mixture of lime and plaster. We introduced into this layer of coating three defects. They have different thicknesses. We finally painted "Al fresco", three bands of paintings, black, green and yellow on the surface of the sample (figure 2).

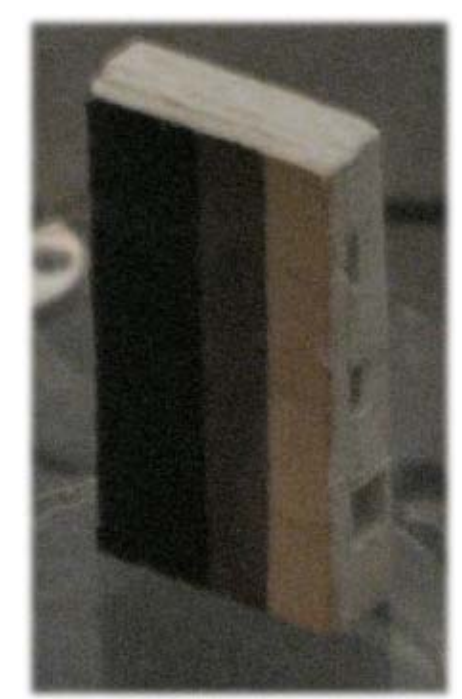

Figure 2: The studied sample

This sample was analyzed on the one hand, using the "PROVISION 100" system. It was analyzed in addition using SAMMTHIR system of the University of Reims. In the first case, the duration of analysis is equal to approximately two seconds. In the second case, the excitation used is of crenel type. Its duration is equal to $10 \mathrm{~S}$. The duration of analysis is equal to $60 \mathrm{~S}$. The power of excitation is equal to $2 * 400 \mathrm{~W}$. Figures 3 present two examples of results obtained. The figure 3a concerns the terahertz analyzes. It shows clearly a more important terahertz signature at the place of the defects. That is due to the absorption of the terahertz waves by the inclusion of air contained in the defects. It shows then, the possibilities of the terahertz method for the detection of this type of defect. It shows that the terahertz method is not susceptible to the different pigments of the pictorial layer too. It is an important advantage. Indeed, compared to the photothermal analysis, the terahertz method excludes a cause of artefact of detection (figure 3b). It shows finally that this experimental configuration, the terahertz method, does not seem sensitive to the thickness of the defect. 


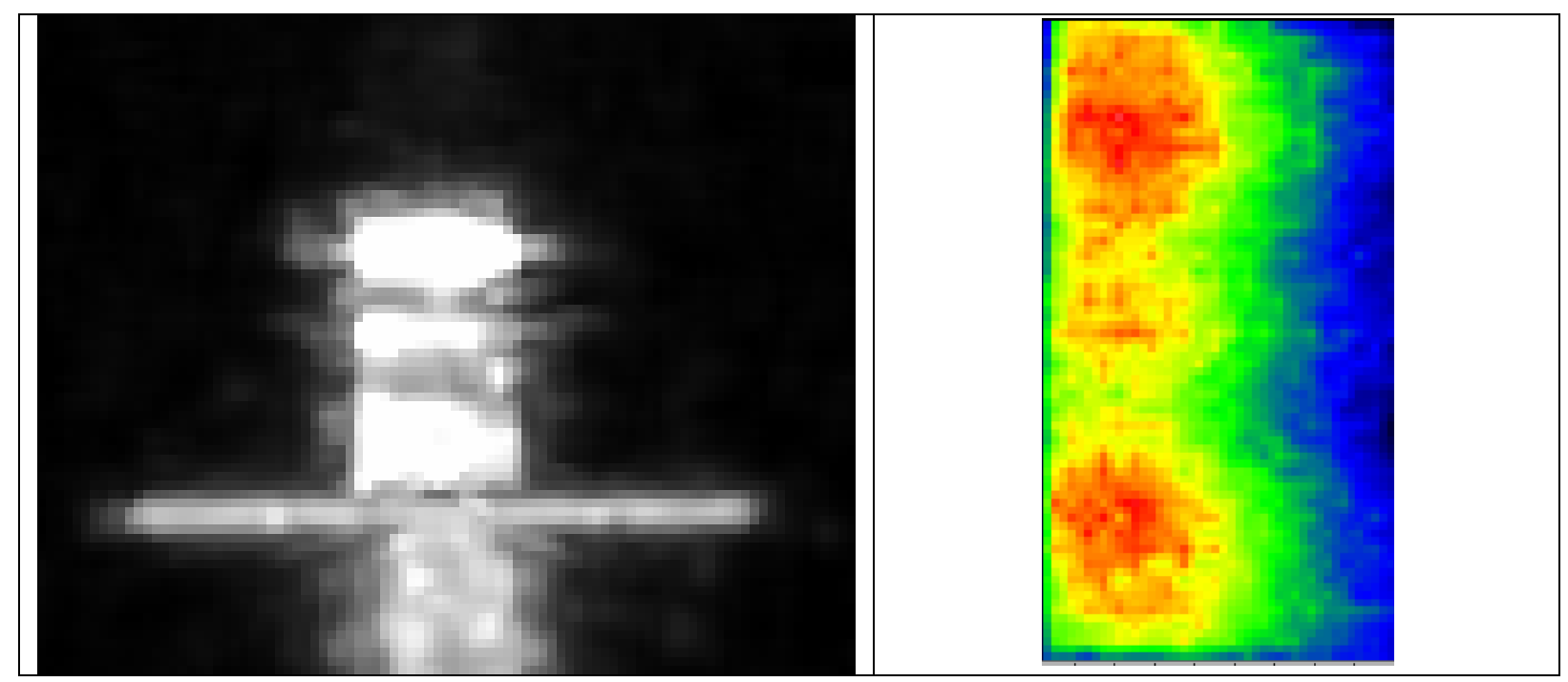

Figure 3: The terahertz (on the left) and photothermal (on the right) results obtained

\section{2) Detection of inclusions located in an academic fresco}

Following these encouraging results, we studied in a second stage, an academic fresco. The latter was made, like the preceding sample. It is according to the primitive Italians technique. A plaster substrate was covered with a mixture of lime and plaster. The whole was then covered with a pictorial layer. It was made "Al fresco". It represents the Jesus child. It is a partial copy of the "Saint Christophe" of the Campana collection of the Louvre (figure 4a). In order to test the possibilities of the terahertz method for the detection of defects located at various depths, it contains five inclusions of plastazote. The defect located in the left and in the lower part of the fresco have a depth equal to $3 \mathrm{~mm}$. The defect located in the right and in the upper part of the fresco have a depth equal to $5 \mathrm{~mm}$. The defect located in the right and in the lower part of the fresco is tilted compared to surface. Its depth varies between $3 \mathrm{~mm}$ and $10 \mathrm{~mm}$. Finally, the defects located in left and in the upper part of the fresco are stacked. The depth of the first one is equal to $3 \mathrm{~mm}$. The depth of the second one is equal to $10 \mathrm{~mm}$. It is thus partially masked by the precedent (figure $4 \mathrm{~b}$ ).

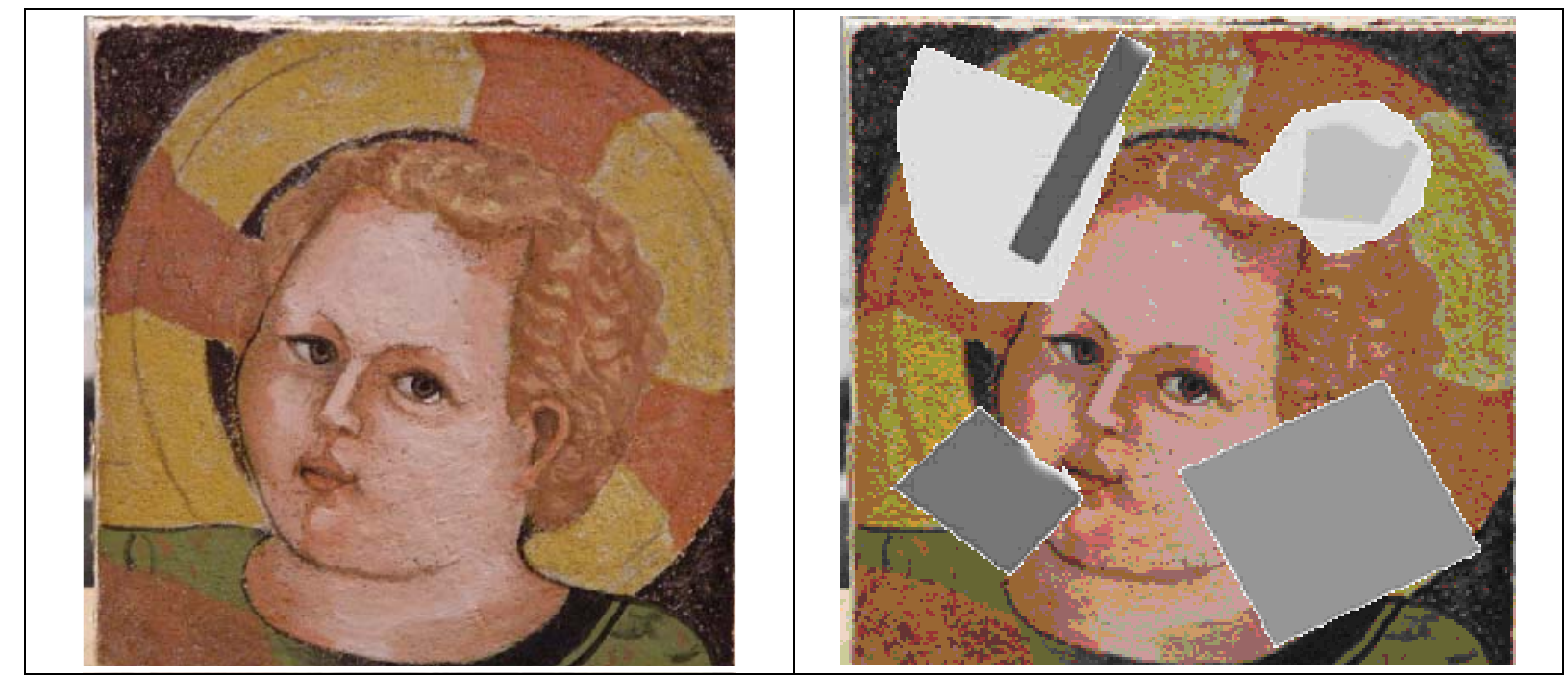

Figure 4: The studied academic fresco (on the left) and the position of inclusions of plastazote (on the right) 
As previously, this academic fresco was analyzed using "PROVISION 100" and "SAMMTHIR" systems. In the first case, the duration of analysis is equal to $2 \mathrm{~s}$. In the second case, the form of the excitation is a crenel type. Its duration is equal to $120 \mathrm{~s}$. The duration of analysis is equal to $300 \mathrm{~s}$. The power of excitation is equal to $500 \mathrm{~W}$. Figures 5 present two examples of results obtained. The figure $5 \mathrm{a}$, concerns the terahertz analysis térahertz. It shows first five characteristic signatures located perpendicularly of the defect. That confirms on the one hand, the possibilities of the terahertz method for the detection of defects located in works of art. That shows in addition that the terahertz method allows the detection of defects located at various depths (up to $10 \mathrm{~mm}$ of depth for the studied sample). That shows finally, that the terahertz method allows also the detection of a defect partially masked by another defect. The figure $5 \mathrm{~b}$ shows that this defect is not detected by the photothermal method. That shows the interest of the terahertz analysis. The figure 5a shows then that the terahertz answer is insensitive with the colours of the pictorial layer. It is not the case of the photothermal method (figure 5b). It is another advantage of the terahertz method. Figures 5 show finally, that the two methods give signature form close to the real geometrical forms of inclusions of plastazote.

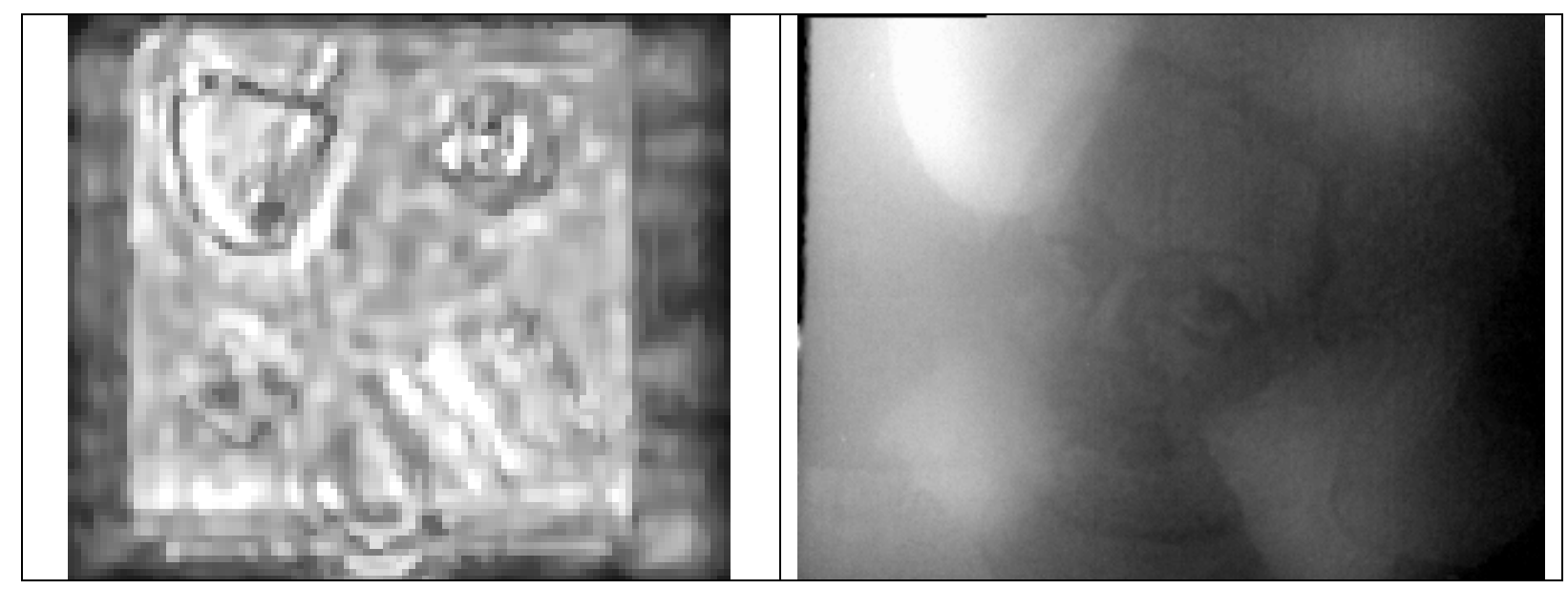

Figure 5: The terahertz (on the left) and photothermal (on the right) results obtained

\section{3) Detection of delamination and galleries of worms in a marquetry.}

The last sample studied is marquetry. It represents a chess-board (figure 6a). This sample is initially made of a piece of plywood. It is covered with piece of oak or piece of beech. In the first layer of plywood, we removed local pieces of wood (figure 6b). That led on the one hand, with the realization of rectangular inclusions of air of various surface extents (8 defects). That led in addition to the realization of fine inclusions of air aiming at simulating galleries of insects (3 defects). This study aimed at approaching in a comparative way, the possibilities of the terahertz method for the detection of low-size defects. 


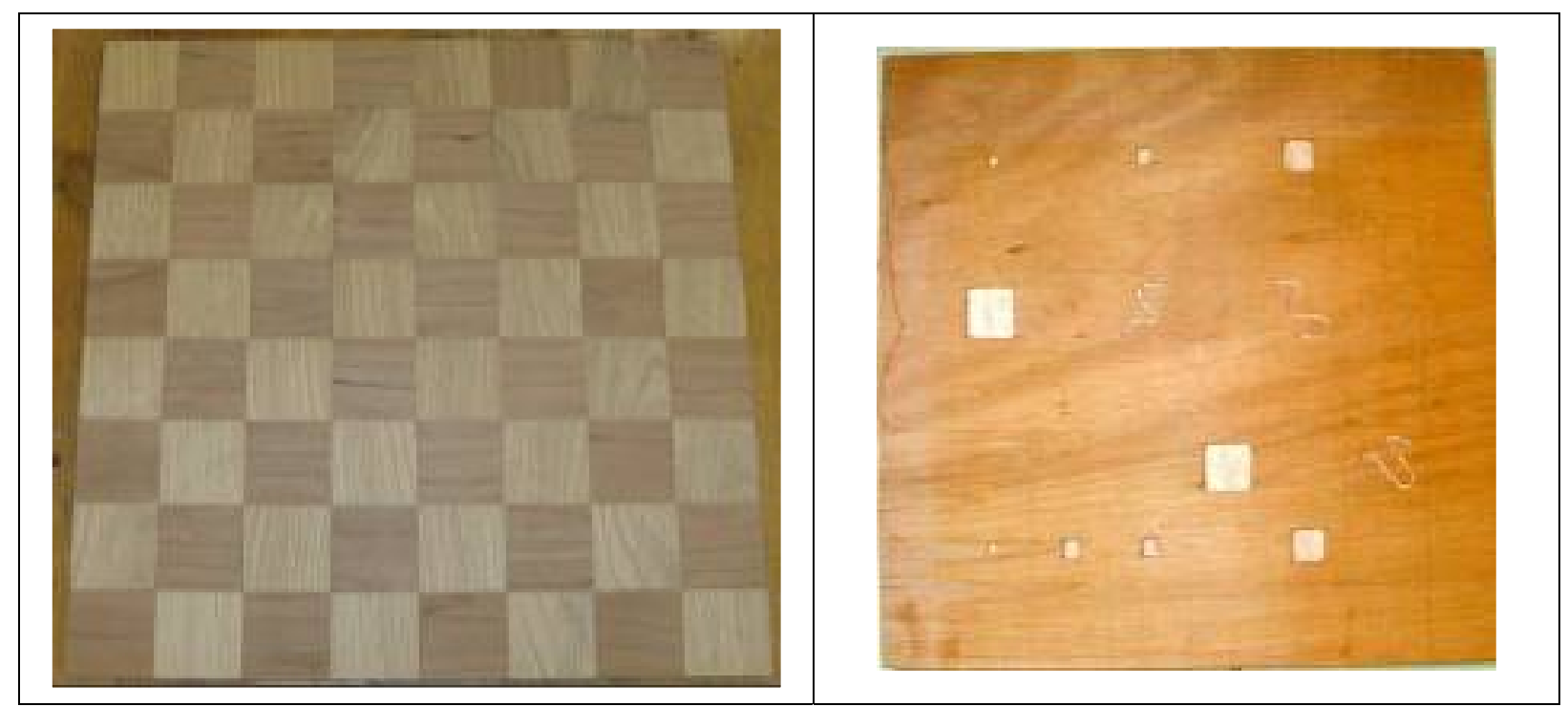

Figure 6: Analyzed marquetry and the position of the delaminations and of the galleries of worms

As previously, this academic sample was analyzed using "PROVISION 100" and "SAMMTHIR" systems.In the first case, the duration of analysis is equal to $2 \mathrm{~s}$. In the second case, the duration of the crenel is equal to $30 \mathrm{~s}$. The power of luminous excitation is equal to 1000 Watts. The duration of the analysis is equal to 512 seconds. Figures 7 present two examples of results obtained. The figure $7 \mathrm{a}$, concerns the terahertz analysis. It reveals characteristic signatures at the perpendicularly of the defects with important geometrical sizes (delamination). That shows the possibilities of the terahertz method for the detection of these types of defects. It also shows that the terahertz method does not allow the detection of the galleries of worms. It is a disadvantage of the terahertz method. It can't permit the detection of low-size defects. This limitation is not met by the photothermal method (figure 7b).

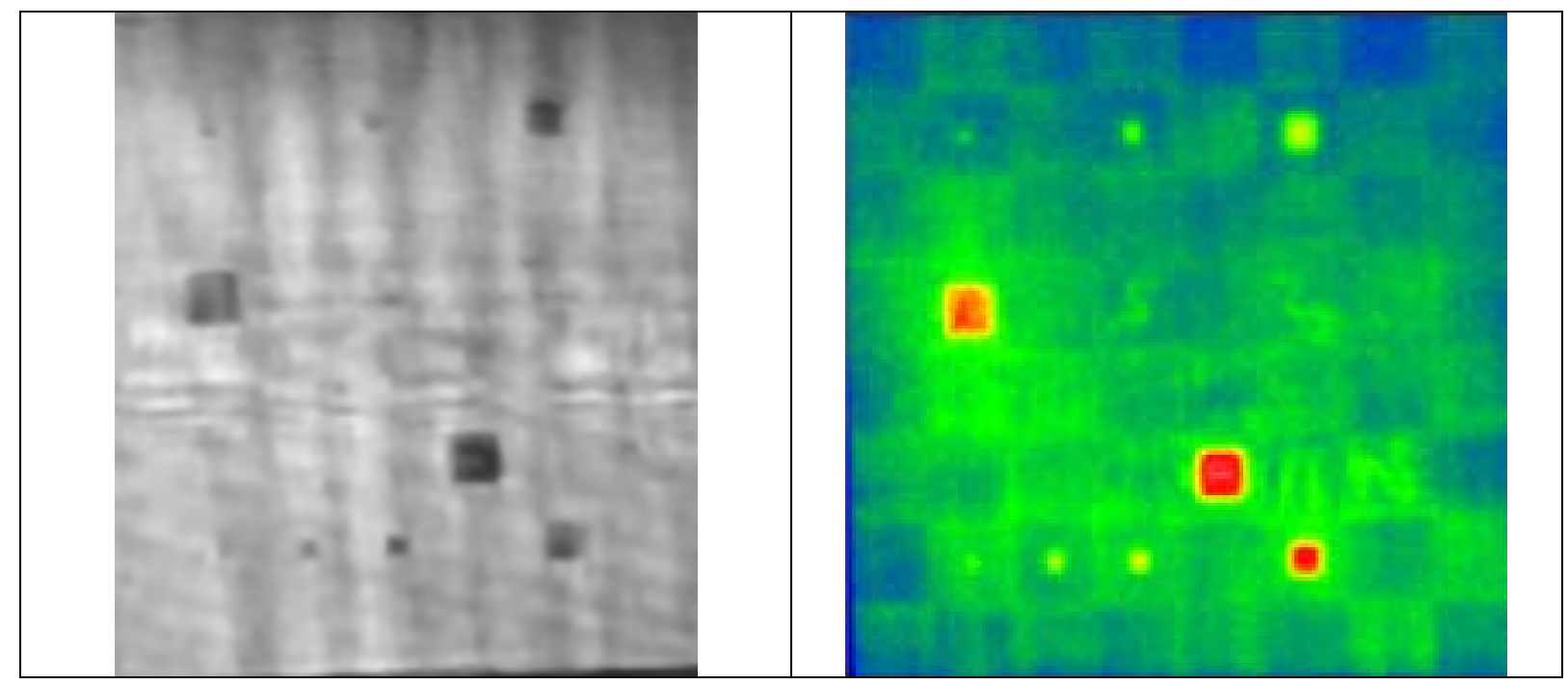

Figure 7: The terahertz (on the left) and photothermal (on the right) results obtained

\section{Conclusion}

In this work, we approached in a comparative way with stimulated infra-red thermography, the possibilities of the terahertz waves for the assistance with the restoration of the works of 
art. This method is non destructive, penetrating and none ionizing. It is serious advantages for this non destructive testing method.

Using industrial "PROVISIOM 100" system of "VISIOM" company, we then showed on the one hand that the method seems to allow the detection of delamination located in murals paintings or in marquetries.

We showed in addition, that the method seems to allow the detection of defects located relatively deeply $(10 \mathrm{~mm})$

We showed in the third time that the method seems to allow the detection of defects potentially masked by other defects. It was not possible by stimulated infra-red thermography.

We showed in the fourth time, that the method does not seem sensitive to the various pigments constituting the pictorial layer. It is an advantage compared to the photothermal method.

We finally showed that the terahertz method was limited for the detection of low-size defects. This limit was not meet by stimulated infra-red thermography.

In conclusion, it seems possible to say that the terahertz method presents some advantages for the assistance of restoration of works of art. Compared to the photothermal method, it is faster, more penetrating and not very sensitive to the pictorial layer. It thus seems of an extremely promising employment in the field of the conservation of the cultural inheritance.

On the other hand, the terahertz method is not very portable. It is expensive. It is limited for the low-size defects detection. It is limited for the quantitative analyses too. Stimulated infra-red thermography does not present by this type of disadvantages. The photothermal method thus remains of a very interesting employment in the field of the restoration of the works of art.

Now, we have to generalize these studies. We have to test this method with "in situ" analyzes. Finally, we have to try to associate the advantages of the two methods of analyses and try to implement them in a coupled way. Studies going in this direction are in progress.

\section{Références bibliographiques}

[1] P. Mounaix : Le nouveau monde des térahertz, Pour la science, mars 2010, n³89, pp 26-32.

[2] F. Oliveira, R. Barat : Analysis of terahertz spectral images of explosives and bio-agents using trained neural networks, SPIE, 5411, 2011.

[3] N. Raimondo, M. Gavalli, Q. Xu, R. Scopigno : Terahertz imagning and non disruptive inspection: A practical case, IRMMW - THZ, 2011.

[4] K. Kawase, Y.Ogawa, Y. Watanabe : Non destructive terahertz imaging of illicit drigs using spectral fingerprints, opt; 2003.

[5] D. Zimdars, J.S. White, G. Stuk, A. Chemovsky, G. Fichter, S. Williamson : Large area terahertz imaging and non destructive evaluation applications, INSIGHT, Non destructive Testing, vol $48, n^{\circ} 9$, sept 2006.

[6] C. Jansen, S. Wietzke, O. Peters, M. Scheller, N. Vieweg, M. Salhi, N. Krumboltz, C. Jordens, T. Hochrein, M. Koch : Terahertz imaging : Applications and perspectives, applied optics, vol 49, 19, pp $48-57,2010$.

[7] Gabriela Szantanik : Etude et restauration d'une peinture murale représentant le Saint Christophe : Mise en œuvre de la radiométrie photothermique pour l'examen du support, INP, Sept 2004.

[8] J.C. Candoré : Détection et caractérisation de défauts par thermographie infrarouge stimulée : Application au contrôle d'œuvres d'art, thèse de doctorat de l'université de Reims, 12 juillet 2010.

[9] Jean Charles Candoré, J.L Bodnar, Vincent Detalle, Philippe Grossel : Non destructive testing of work of art by stimulated infrared thermography, European physical journal Applied Physics, fev 2012, vol 57, 02, pp 21002 -210011.

[10] Jean Charles Candoré, J.L Bodnar, Vincent Detalle, Philippe Grossel : Characterization of defects situated in a fresco by stimulated infrared thermography, European physical journal Applied Physics, jan 2012, vol 57, 01, pp 11002 -11008. 